\title{
ICRH physics and technology achievements in JET-ILW
}

P. Jacquet ${ }^{1, *}$, D. Van Eester ${ }^{2}$, E. Lerche ${ }^{2,1}$, V. Bobkov ${ }^{3}$, T. Blackman ${ }^{1}$, L. Colas ${ }^{4}$, C. Challis ${ }^{1}$, A. Czarnecka ${ }^{5}$, P. Dumortier $^{2,1}$, D. Frigione ${ }^{6}$, F. Durodié ${ }^{2}$, L. Garzotti ${ }^{1}$, M. Goniche ${ }^{4}$, J. Graves ${ }^{7}$, Y. Kazakov ${ }^{2}$, K. Kirov ${ }^{1}$, C. C. Klepper ${ }^{8}$, N.

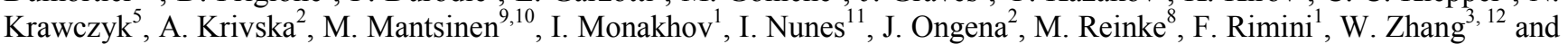
JET contributors ${ }^{13}$

EUROfusion Consortium, JET, Culham Science Centre, Abingdon, OX14 3DB, UK.

${ }^{1}$ CCFE, Culham Science Centre, Abingdon, OX14 3DB, UK.

${ }^{2}$ LPP-ERM/KMS, EUROfusion Consortium Member, TEC Partner, Brussels, Belgium.

${ }^{3}$ Max-Planck-Institut für Plasmaphysik, Boltzmannstr.2, D-85748, Germany.

${ }^{4}$ CEA, IRFM, F-13108 Saint-Paul-lez-Durance, France.

${ }^{5}$ IPPLM, Hery 23 Str., 01-497 Warsaw, Poland.

${ }^{6}$ Associazione ENEA, Fusion and Nuclear Safety Department, C. R. Frascati, Via E. Fermi 45, 00044 Frascati (Roma), Italy.

${ }^{7}$ Centre de Recherches en Physique des Plasmas, EPFL, 1015 Lausanne, Switzerland.

${ }^{8}$ Oak Ridge National Laboratory, PO Box 2008, Oak Ridge, USA.

${ }^{9}$ Barcelona Supercomputing Center (BSC), 08034 Barcelona, Spain.

${ }^{10}$ ICREA, Pg. Lluis Companys 23, 08010 Barcelona, Spain.

${ }^{11}$ Instituto de Plasmas e Fusão Nuclear, IST, Universidade de Lisboa, Portugal.

${ }^{12}$ Department of Applied Physics, University Ghent, 9000 Gent, Belgium

${ }^{13}$ See the author list of "Overview of the JET results in support to ITER" by X. Litaudon et al. to be published in Nuclear Fusion Special issue: overview and summary reports from the 26th Fusion Energy Conference (Kyoto, Japan, 17-22 October 2016).

\begin{abstract}
ICRH was extensively used in the 2015-16 JET-ILW (ITER like wall) experimental campaign; bulk heating together with high-Z impurity chase-out from plasma centre importantly contributed to the good DD fusion performance obtained recently in JET. Power up to $6 \mathrm{MW}$ was launched in H-mode deuterium plasmas and $8 \mathrm{MW}$ during the hydrogen campaign. The ILA was re-installed and contributed positively to the availability of ICRH power. The ILA produces slightly less high-Z impurities than the A2's and the PWI measured via Be line emission on limiters is in the same ballpark. Specific experiments were conducted to optimise ICRH scenarios in preparation for DT in particular the dual frequency scheme, $(\mathrm{H}) \mathrm{D}$ and $(\mathrm{He}) \mathrm{D}$ were tested. In addition, it was confirmed that the (D)H scenario is accessible in a ILW environment and the novel 3-ions ICRH scheme was validated experimentally.
\end{abstract}

\section{Introduction}

In 2015-16, JET experiments were run to develop plasma scenarios in preparation for the DT campaign. ICRH was routinely used in plasma scenario development. ICRH provides bulk plasma heating and a localised central heat source for high$Z$ core impurity screening. The paper describes the progress to reliably and efficiently deliver high ICRH power to the plasma and describes experiments aiming at optimizing ICRH heating scenarios in preparation for the JET DT campaign and ITER non-active phase operation.

\section{Operational aspects}

The JET ICRH system $[1,2]$ includes 4 A2 antennas and the ITER-Like Antenna (ILA) which, after its initial operation in 2008-2009 [3] was re-installed and recommissioned in 2015 [4]. The frequency coverage is 23-57 MHz for the A2's and 29-51 MHz for the ILA. Each A2 antenna is a phased array of 4 poloidal straps; controlling the phase between straps allows waves to be

\footnotetext{
Corresponding author: philippe.jacquet@ukaea.uk
} 

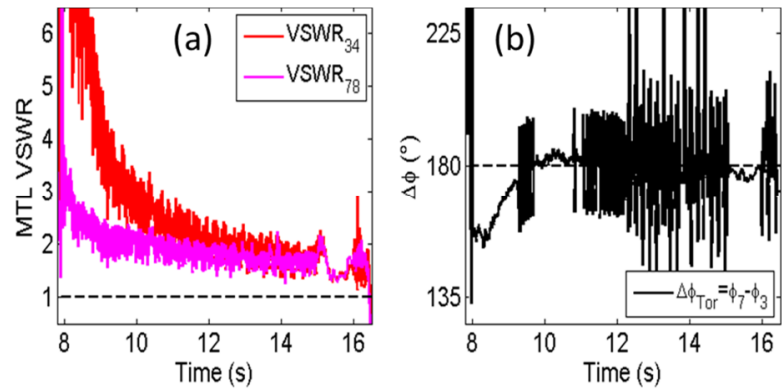

Fig. 2. Reproduced from [4], illustration of ILA real time matching, JPN 91916 (ELM-y H-mode), bottom row only is used. (a) VSWR in the main transmission lines feeding bottom straps 34 and 78 (2nd stage matching); (b) phase between toroidally adjacent straps 7 and 3 .

launched with different $\mathrm{k}_{/ /}$spectra. Usually $[0, \pi, 0, \pi](\pi$ phasing), $[0, \pi / 2, \pi,-\pi / 2](+\pi / 2$ phasing) or $[0,-\pi / 2, \pi$, $\pi / 2](-\pi / 2$ phasing) phasing is applied to the straps [1, 2 , $3,4]$. ELM resilience for the A2 antennas is achieved by pairing the antennas using $3 \mathrm{~dB}$ hybrid couplers (A\&B antenna system) or external conjugate-T's (ECT, C\&D antenna system) (see Fig. 1). The ILA is an array of 8 straps ( 4 in the poloidal direction $\times 2$ in the toroidal direction); neighbour straps in the poloidal direction are paired as Resonant Double Loops (RDL) using invacuum capacitors and T-junctions, which, with the matching system, provide ELM resilience.

The implementation of real-time antenna matching algorithms greatly improved the ILA system availability [4]. Now automated are: the impedance at the RDL's Tjunction, the stub/trombone impedance matching $\left(2^{\text {nd }}\right.$ stage matching) behind the RDL's, the phase between toroidally adjacent RDL's, and the phase between top/bottom rows. Fig. 2 shows an example of real-time matching in an H-mode pulse. In most of the campaign, only one row of the ILA was used (either top or bottom); a fluid leak in a capacitor actuator developed in summer 2016 (stopping then operation of the top array) before all controls for operation of the full array were fully commissioned. Despite this limitation, power levels of up to $2.8 \mathrm{MW}$ were coupled from the ILA $(\sim 1.5 \mathrm{MW}$ in $\mathrm{H}$-mode) and in general the ILA contributed to $\sim 1 / 3$ of the total ICRH power.

Operational aspects for the A2 antennas and the ICRH plant are discussed in details in [5]. When the plasma conditions are such that antenna coupling resistance $\left(\mathrm{R}_{\mathrm{c}}\right)$ is low $\left(\mathrm{R}_{\mathrm{c}}<1.0 \Omega\right.$, typical of H-mode plasmas with low gas fuelling) the power is bounded by the maximum voltage in the transmission lines of $\sim 30 \mathrm{kV}$. Operating above this voltage increases the risk of arcing. However, improving the power balance between straps and between generators is a way to effectively increase the launched power in these low coupling conditions. Statistically, most of the ICRH pulses in JET-ILW were performed with antenna coupling resistance $\mathrm{R}_{\mathrm{c}}>1.0 \Omega$. In this case, the capability of the A2 system to deliver power to the plasma is effectively bounded by the available power from the generators and the ohmic losses. ICRH power gain can be obtained from ICRH generator tuning and maintenance to ensure they operate reliably close to their specifications.
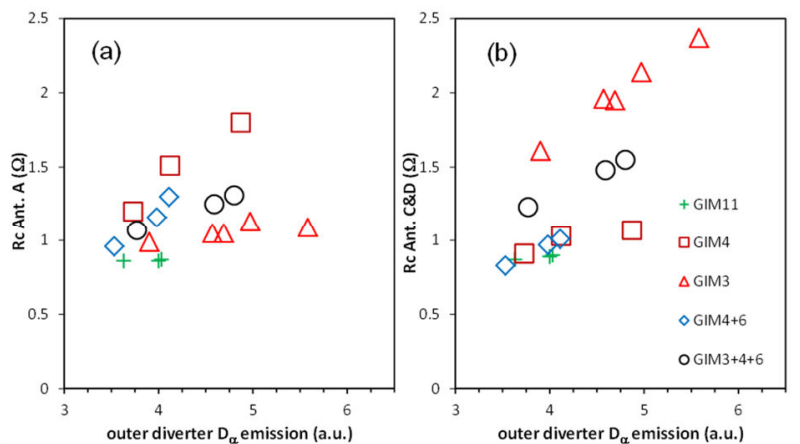

Fig. 3. $\mathrm{R}_{\mathrm{c}}$ vs divertor $\mathrm{D}_{\alpha}$, with divertor or OMP gas injection. (a) antenna A, (b) antennas C\&D. Experimental conditions: ELMy H-mode plasma with $\mathrm{N}=1(\mathrm{H}) \mathrm{D}$ ICRH, $\mathrm{f}_{\mathrm{ICRH}}=42 \mathrm{MHz}$, $\mathrm{B}_{\mathrm{t}}=2.7 \mathrm{~T}, \mathrm{I}_{\mathrm{P}}=2.5 \mathrm{MA}, \mathrm{P}_{\mathrm{NBI}}=15 \mathrm{MW}, \mathrm{P}_{\mathrm{ICRH}}=3 \mathrm{MW}$.

\section{SOL density tailoring to increase ICRH coupling}

As explained in the previous section, increasing $R_{c}$ allows operation away from transmission line voltage limits and maximisation of the ICRH power. The fast wave can only propagate beyond the evanescent layer in the Scrape-Of Layer (SOL). Typically the cut-off density is a few $10^{-18} \mathrm{~m}^{-3}$ in tokamaks with $\mathrm{B}_{\mathrm{T}}=\mathrm{a}$ few Tesla. Since 2007, experiments are carried-out in various tokamaks, including JET, to tailor the SOL density in front of the ICRH antennas and reduce the fast wave evanescence gap [6, 7]. In 2016, a new Outer-Mid Plane (OMP) Gas Injection Module (GIM), was installed between antenna C and antenna D (GIM 3, see Fig. 1), allowing to develop further the gas injection technique to maximise the ICRH power. Fig. 3 illustrates the gain in coupling resistance on antenna $\mathrm{A}$ and antennas C\&D when using gas from GIM 4 (resp. GIM 3) in H-mode plasmas. It is also important to note that using a combination of outer GIMs (for example GIM 3+4+6) allows a substantial increase of $\mathrm{R}_{\mathrm{c}}$ on all JET antennas simultaneously. The effect of local gas injection on JET ICRH antenna coupling was modelled [8] using the 3D EMC3-Eirene SOL modelling. A 3D JET model was built as a realistic simulation model. This model includes all 5 ICRH antennas, the poloidal limiters, the pumped divertor, and all major obstacles for gas motion in the JET chamber. First, a reference pulse from the experiment with symmetrical divertor gas puffing was simulated and in particular, transport parameters were adjusted to match the measured profiles of $n_{e}, T_{e}$ and ion saturation current in the divertor. This validated plasma was then used in the simulations to study the changes in the SOL when puffing gas from a specific GIM location at the OMP or at the top of the vessel, while other parameters were kept constant. No specific ICRH-SOL interaction was taken into account in the simulations; the changes in the SOL are exclusively from the change to the location of the ionisation via plasma electron impact of the neutrals. Simulations in these conditions show that localised OMP gas injection increases the SOL density close to the injection point which results in a shift of few centimetres of the cut-off layer position. Using a simple 1D fast wave coupling code [7], the changes in SOL 


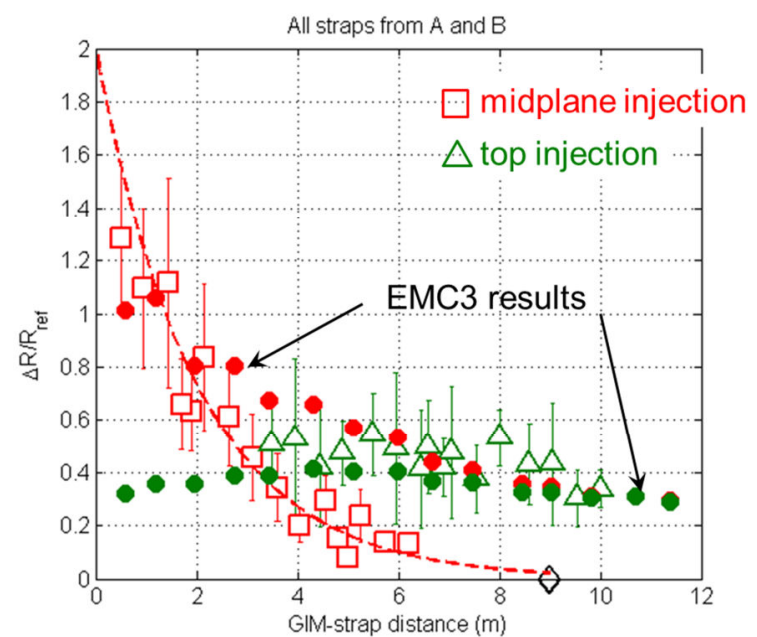

Fig. 4. Reproduced from [8], $\mathrm{R}_{\mathrm{c}}$ improvement w.r.t. divertor gas, measurements and simulations. Open symbols: experimental data. The simulation results are shown with the plain circles (red: OMP injection; green: top injection).

density profiles at different toroidal positions (averaged poloidally over the height of an A2 antenna) were translated in terms of relative $R_{c}$ increase when changing from divertor to top or OMP gas injection. The result is shown in Fig. 4. The simulations can reproduce qualitatively the $\Delta R_{c} / R c$ trend with injection-strap distance indicating that ionisation of the neutrals by the plasma is the main physical process at play in the local increase of density in front of the antennas when using localised gas injection. The JET benchmarking of the EMC3-Eirene simulations against the experimental results also gives confidence that this simulation tool can confidently be used on ITER to assess the effect of localised gas injection on ICRH antenna performance.

\section{Characterisation of ICRH enhanced PWI}

Although ICRH is the main tool used to prevent W accumulation in the centre of JET plasmas, application of ICRH usually leads to an overall increase of the plasma impurity content, and in particular in JET-ILW, tungsten (W) and nickel (Ni). This is in general attributed to an enhanced Plasma Wall Interaction (PWI) and to sputtering of the Plasma Facing Components (PFC) when applying ICRH. It is also known that the rapid variation of the Radio-Frequency (RF) rectified potential across the equilibrium magnetic field can cause significant convective transport $(\mathrm{E} \times \mathrm{B}$ drifts $)$ in the scrape-off layer (SOL) $[9,10]$. One could argue that this $\mathrm{RF}$ driven SOL transport, can enhance the penetration of impurities from the SOL to the plasma core. Experiments were carried out in JET to assess this latter effect [11]. By injecting a fixed amount of the $\mathrm{N}_{2}$ molecules from single location in L-mode discharges and toggling the ICRF power between the antennas, the influence of the proximity of the $\mathrm{N}_{2}$ injection to the active antennas on the core $\mathrm{N}$ content was characterized via measurement of the N VII spectral line intensity. No significant influence of proximity of the active antenna on the core $\mathrm{N}$ content was observed when toggling the
ICRF power between antennas $\mathrm{A}+\mathrm{B}$ (close to $\mathrm{N}_{2}$ injection) and D (away). This is independent of the order of the antennas. A similar result is obtained when using $\pi,-\pi / 2$, or $+\pi / 2$ strap phasing. These observations show that proximity of a powered antenna close to an impurity source does not significantly affect penetration of these impurities, ruling out the hypothesis that RF driven changes in edge transport plays a significant role in the enhanced impurity content when using ICRH.

The exact location of the RF driven impurity sources is still unknown, as is the exact release mechanism(s). In particular, there is no tungsten or nickel PFCs close to the ICRH antennas; the W-I line emission on tungsten PFCs (for example, divertor baffles, inner wall NBI shine-through protection) [12], did not show evidence of enhanced W sputtering when using ICRH. However RF sheath rectification [13] is suspected to play a significant role. In the past, SOL density modifications at locations magnetically connected few meters away from active ICRH antennas have been evidenced [14]. It is well possible that given the size of the antennas, a large area of tungsten (for example divertor baffles) is experiencing enhanced sputtering, however below spectroscopy diagnostic sensibility level. Experiments were recently conducted to address the question of the impact of the antenna geometry on RF induced PWI. First, an experiment was conducted to compare Be sputtering on objects connected either to a conventional A2 antenna or connected to the ILA antenna [15]. In L-mode plasmas, Be II line emission signal at the outer poloidal limiter locations 'D14' and 'K14' was monitored when the ICRH power was toggled between different antennas:
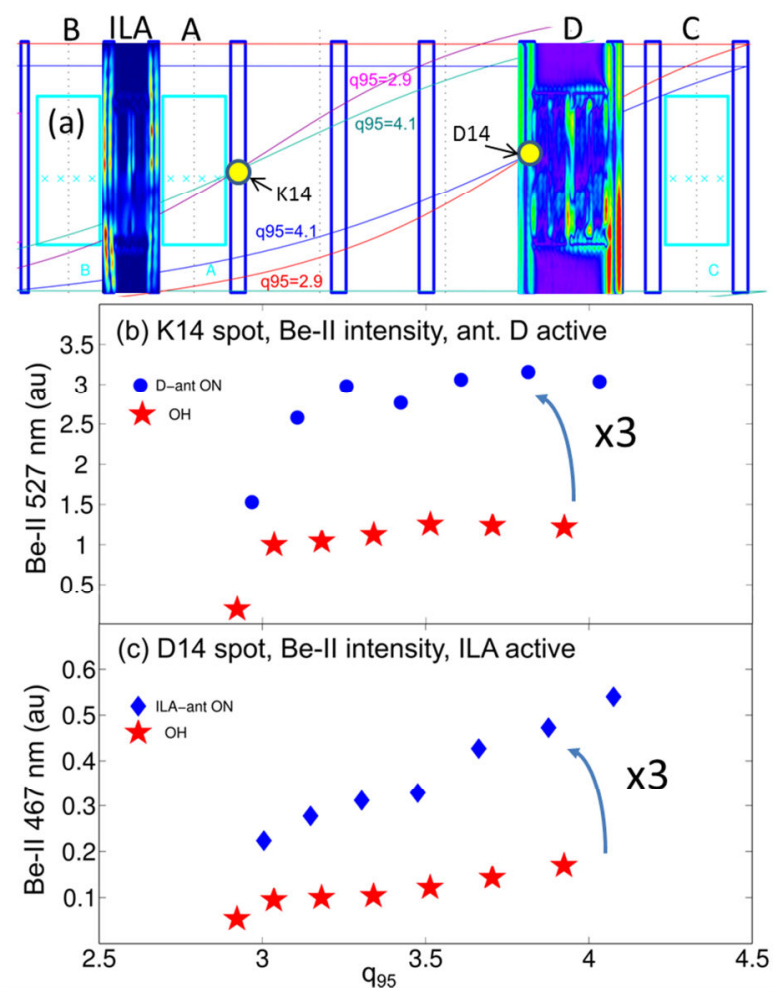

Fig. 5. Reproduced from [15], (a) field lines from K14 and D14 points at two instants of JPN 90554 with $\mathrm{q}_{95}=2.9$ and $\mathrm{q}_{95}=4.1$. Be II line emission measurement at K14 (b) and D14 (c) with resp. D and ILA active. $0.5 \mathrm{MW}$ per antenna and $-\pi / 2$ strap phasing was used. 


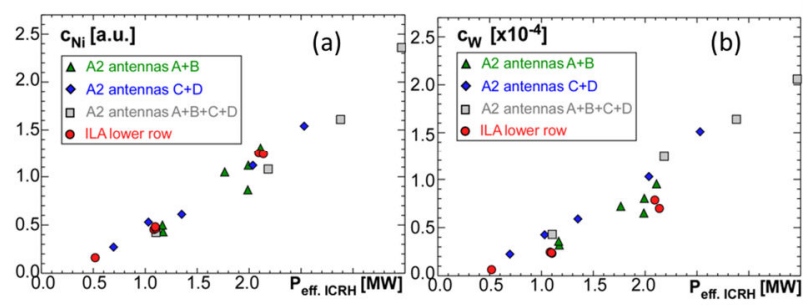

Fig. 6. JET plasma impurity content computed at plasma midradius from VUV spectroscopy vs ICRH power when using the ILA, A\&B, C\&D or all the A2 antennas, (a) Nickel and (b) Tungsten.

A\&B, D, or ILA. D14 intercepts field lines passing few centimetres in front of the bottom of the ILA limiter while K14 intercepts field lines passing few centimetres in front of the top of antenna D limiter. In these pulses, $\mathrm{q}_{95}$ was changed so as to scan the altitude of the field lines in front of the ILA (resp. D) limiters probed by D14 (resp. K14) measurement. A typical result is on Fig. 5. The same level of Be line emission increase (w.r.t. the ohmic level) is observed when probing in front of the active ILA or active antenna D limiters. Further analysis are ongoing to verify if these observations are coherent with sputtering models (using the ERO code [16]) and combined antenna /RF sheath rectification models [17] (using the TOPICA antenna code [18] and the SSWICH code [19]).

Secondly, the impact of using antennas with different geometries on core impurity content (W and $\mathrm{Ni}$ ) was studied in a series of ICRH L-mode pulses where different combinations of antennas were energised [11]. Fig. 6 summarises the results. The $\mathrm{Ni}$ and $\mathrm{W}$ concentration are in the same ballpark for all combinations of antennas used, the ILA producing slightly less $\mathrm{W}$ and radiation when looking at a larger database. As a next step in the analysis, we will compare $\mathrm{E}_{/ /}$maps calculated by TOPICA and the rectified potentials modelled by SSWICH for the two antennas.

\section{ICRH scenarios for JET DTE2}

Recently, record DD fusion performances for JET-ILW plasmas were obtained; ICRH was extensively used in the baseline (proxy for the $\mathrm{Q}=10$ ITER reference scenario with $\mathrm{I}_{\mathrm{p}}=15 \mathrm{MA}$ at $\mathrm{q}_{95} \sim 3$ ) and hybrid (proxy for the $\mathrm{Q}=5 \mathrm{t}>1000 \mathrm{~s}_{\mathrm{p}}=12 \mathrm{MA}$ at $\mathrm{q}_{95} \sim 4.3$ ITER scenario) experiments aiming at preparing plasma scenarios for the future (2018-19) JET D-T campaign; this is illustrated for baseline pulses in Fig. 7. Reference [20] also reports on the use of ICRH in the hybrid scenario. Several factors contributed to the recent record performances, in particular, the availability of high power NBI and ICRH, and operation with reduced gas dosing. This latest condition has two implications: (i) discharges with lower gas dosing are more prone to impurity accumulation, then ICRH became a key ingredient, providing central heating and tungsten chase-out from the centre, to ensure stability of these discharges [21,22]; (ii) as the total gas dosing was reduced, OMP GIMs must be used to maximise ICRH power (see previous section). However some questions remain to be addressed in the context of D-T plasmas. The 'reference' ICRH scenario for D-T is

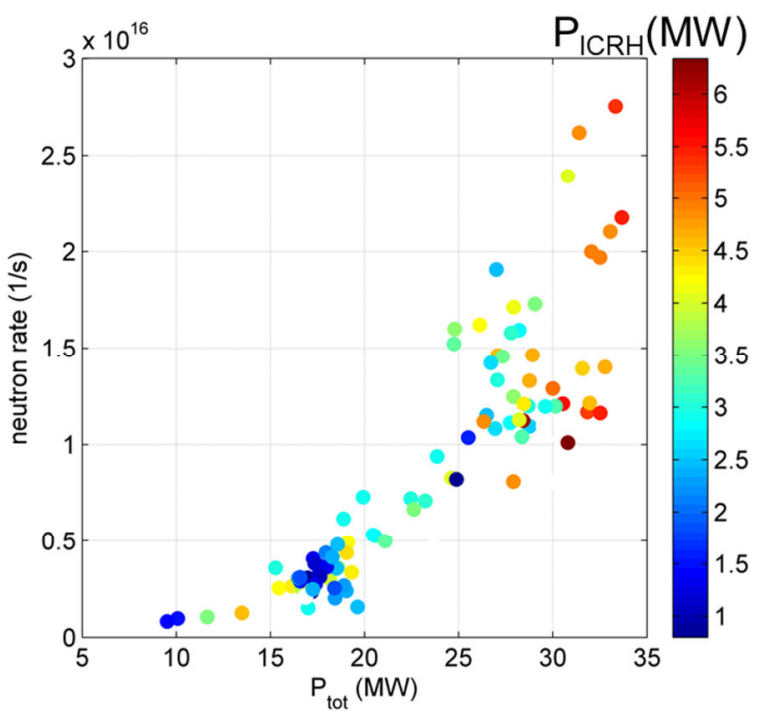

Fig. 7. For baseline scenario plasmas, neutron yield vs $\mathrm{P}_{\text {tot }}=\mathrm{P}_{\mathrm{NBI}}+\mathrm{P}_{\mathrm{ICRH}}$ power. The data points are colour coded with the ICRH power, the $\mathrm{N}=1(\mathrm{H}) \mathrm{D}$ scenario was used. The data base includes JET campaign C36 pulses with Ip $>2.5$ MA.

$\mathrm{N}=2 \mathrm{~T}$ heating assisted by $\mathrm{N}=1$ minority ${ }^{3} \mathrm{He}$ heating; this scenario maximises ion heating and hence has the potential to boost fusion power [23]. However, some authors have emphasized the role of central electron heating that, aside from providing peaked temperature profiles, increases turbulent transport to prevent tungsten accumulation [24]. In this context the effectiveness of minority ${ }^{3} \mathrm{He}$ heating to chase-out $\mathrm{W}$ from plasma centre needs to be characterised. One can also envisage a scenario where hydrogen minority and ${ }^{3} \mathrm{He}$ minority are combined to act on $\mathrm{W}$ accumulation and fusion performance at the same time. Some experiments were recently carried out to compare different ICRH schemes in similar $2.7 \mathrm{~T} / 2 \mathrm{MA} \mathrm{H}$-mode discharges with $\mathrm{P}_{\mathrm{NBI}} \sim 15$ $\mathrm{MW}$ and $\mathrm{P}_{\mathrm{ICRH}}$ in excess of $6 \mathrm{MW}$ : $(\mathrm{H}) \mathrm{D}$ heating with
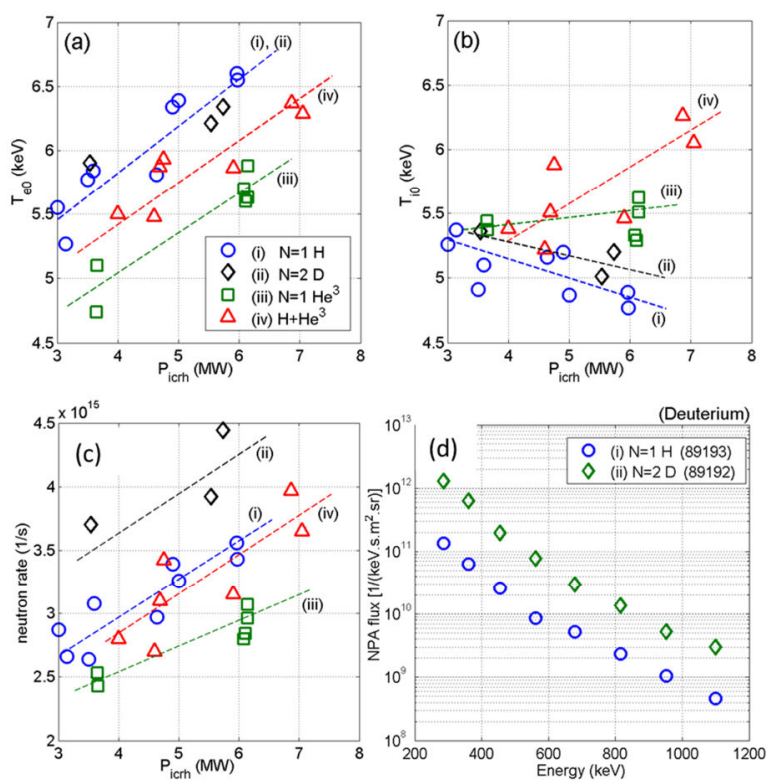

Fig. 8. Summary of experiments to compare different ICRH scenarios: $\mathrm{N}=1 \mathrm{H}$ minority (and $\mathrm{N}=2 \mathrm{D}$ heating when $\% \mathrm{H}$ is low), $\mathrm{N}=1{ }^{3} \mathrm{He}$ minority, and combined heating. (a) $\mathrm{Te}$, (b) $\mathrm{Ti}$ from CXS, (c) neutron yield. On (d) NPA measurement for JPN $89192(\% \mathrm{H}<1 \%)$ and $89193(\% \mathrm{H}=2.5 \%)$ are compared. 
$\mathrm{f}_{\mathrm{ICRH}}=51 \mathrm{MHz},\left({ }^{3} \mathrm{He}\right) \mathrm{D}$ heating with $\mathrm{f}_{\mathrm{ICRH}}=32.5 \mathrm{MHz}$, and the combined heating scheme where both minorities are present in the plasma and the ICRH power is split between $51 \mathrm{MHz}$ and $32.5 \mathrm{MHz}$. This was the first time this combined heating scenario was characterised, in particular we could demonstrate safe operation of $(\mathrm{H}) \mathrm{D}$ ICRH with the presence of the $\mathrm{N}=1{ }^{3} \mathrm{He}$ resonance at the JET inner wall.

A summary of these experiments is shown in Fig. 8. As expected, electron temperature was maximised when using (H)D ICRH; ion temperature was maximised when using the combined heating scheme. It must be emphasized that in the $\left({ }^{3} \mathrm{He}\right) \mathrm{D}$ heating pulses the ${ }^{3} \mathrm{He}$ concentration was $<2 \%$ which is too low since the optimum RF power absorption is expected to take place for $\%{ }^{3} \mathrm{He} \sim 7 \%$ [25]. The combined heating pulses which show a better ion heating were run with $\%{ }^{3} \mathrm{He} \sim 3.5 \%$. Because of the non-optimal conditions for $\%^{3} \mathrm{He}$ in the pure $\left({ }^{3} \mathrm{He}\right) \mathrm{D}$ heating pulses, no conclusion could be drawn about the effectiveness of this scenario to prevent $\mathrm{W}$ accumulation. Also, future similar experiments should ideally be done in conditions where, without ICRH, plasma is unstable due to $\mathrm{W}$ accumulation.

Also noticeable in Fig. 8 is the family of points where all the ICRH power was used with $\mathrm{f}_{\mathrm{ICRH}}=51 \mathrm{MHz}$ and with the hydrogen concentration reduced from $\sim 2.5 \%$ ( $\bigcirc)$ to less than $1 \%(\diamond)$ In this case, $N=2 \mathrm{D}$ heating plays a significant role, creating a population of energetic D ions leading to a $25 \%$ increase of the neutron yield w.r.t the (H)D standard case. This is supported by D fast ions measurements from the Neutral Particle Analyser and neutron time of flight spectroscopy (TOFOR) [25]. Similar observations were made in recent hybrid scenario pulses [20].

\section{ICRH scenarios for JET H campaign and ITER non-active phase}

The path towards the JET D-T campaign includes

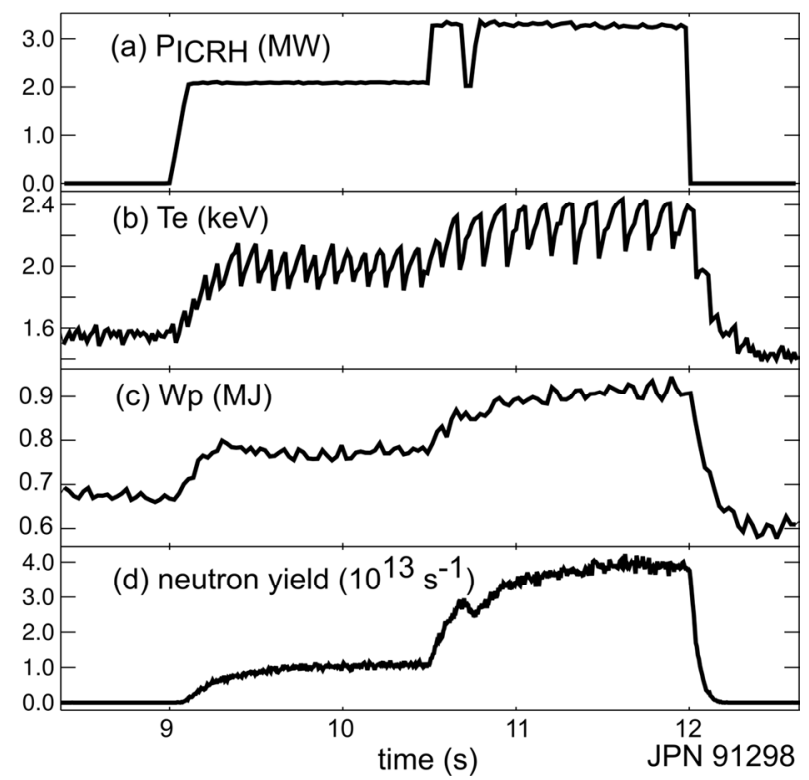

Fig. 9. Example of $\mathrm{N}=1$ (D)H ICRH. (a) ICRH power, (b) central electron temperature, (c) MHD plasma energy, (d) neutron yield. The $\mathrm{D}$ concentration is this pulse was $\sim 1.5 \%$
Hydrogen and Tritium campaigns to characterise isotope effects on confinement properties and $\mathrm{H}$-mode threshold in which ICRH is needed. It is also important to test the ICRH scenarios considered for the ITER non-active phase [26] in the ILW environment, and validate the modelling codes used to predict ICRH absorption in ITER. Some of these scenarios were never tested experimentally (the 3-ion scheme) or were tested in unfavourable conditions ( $\mathrm{N}=1$ (D)H in JET-C). The following summarises the ICRH scenarios used or tested during the JET 2016 hydrogen campaign.

- $\mathrm{N}=2 \mathrm{H}$ majority, with $\mathrm{B}_{\mathrm{T}} \sim 1.8 \mathrm{~T} / \mathrm{f}_{\mathrm{ICRH}}=51 \mathrm{MHz}$, applicable for ITER $1 / 3$ field non-active phase with the same $B_{T} / f_{I C R H}$ parameters: this scenario was extensively used in H-mode access experiments at low field and to characterize low activation $\mathrm{p}+\mathrm{Be}$ nuclear reactions [27]; the ICRH aspects are reported in [28].

- $\mathrm{N}=1\left({ }^{3} \mathrm{He}\right) \mathrm{H}$ with $\mathrm{B}_{\mathrm{T}} \sim 3.2 \mathrm{~T} / \mathrm{f}_{\mathrm{ICRH}}=32.5 \mathrm{MHz}$, applicable for the ITER full-field non-active phase with $\mathrm{B}_{\mathrm{T}}=5.3 \mathrm{~T} / \mathrm{f}_{\mathrm{ICRH}}=53 \mathrm{MHz}$ : this scenario was extensively used in the H-mode access experiments at higher field and in transport studies [29]. A recent report on this ICRH scheme is given in [30]. The heating efficiency evaluated using the brake-in slope analysis technique was $\mathrm{P}_{\mathrm{abs}} / \mathrm{P}_{\text {in }} \sim 70 \%$ and heating performances of $\sim 0.14$ $\mathrm{MJ} / \mathrm{MW}\left(\mathrm{I}_{\mathrm{P}}=2.5 \mathrm{MA}\right)$ to $\sim 0.11 \mathrm{MJ} / \mathrm{MW}\left(\mathrm{I}_{\mathrm{P}}=1.8 \mathrm{MA}\right)$ were observed (note that the heating performance critically depends on plasma energy transport properties and hence on details of the experimental conditions). In this so called 'inverted scenario' it is important that the minority concentration is controlled to the optimal value of $\sim 2 \%$. At too small minority concentration, the heating degrades while at larger concentrations the mode conversion regime is entered and direct electron heating comes more significantly into play.

- $\mathrm{N}=1$ (D)H with $\mathrm{B}_{\mathrm{T}} \sim 3.3 \mathrm{~T} / \mathrm{f}_{\mathrm{ICRH}}=25 \mathrm{MHz}$; the parent $\left({ }^{4} \mathrm{He}\right) \mathrm{H}$ is applicable for the ITER full-field non-active phase with $B_{T}=5.3 T / f_{I C R H}=41 \mathrm{MHz}$. This heating scenario was not accessible on JET-C because the concentration of carbon $(2-3 \%$ ) (carbon has the same $\mathrm{Z} / \mathrm{A}=6 / 12$ as deuterium) was such that a mode-conversion layer was located on the low field side of the D resonance layer [31]. In JET-ILW the heating scheme becomes available as the carbon concentration is a fraction of percent and $\mathrm{Be}$, the main low $\mathrm{Z}$ impurity has $\mathrm{Z} / \mathrm{A}=4 / 9$. Fig. 9 summarises the results of preliminary tests for this heating scheme performed in the last campaign. Plasma heating is indeed now observed; the heating efficiency was $\sim 45 \%$ and the plasma performance was $\sim 0.1$ $\mathrm{MJ} / \mathrm{MW}\left(\mathrm{I}_{\mathrm{p}}=2 \mathrm{MA}\right)$. The increase of the neutron yield and the detection of a fast deuterium tail by the TOFOR diagnostic (not shown) are other indications of D minority heating. Further experiments are planned to optimise this scheme, as the condition for maximum wave absorption, expected for $\% \mathrm{D} \sim 3 \%$, was not reached.

- The 3-ion scheme [32] D- $\left({ }^{3} \mathrm{He}\right)-\mathrm{H}$ with $\mathrm{B}_{\mathrm{T}} \sim 3.2 \mathrm{~T}$ and $\mathrm{f}_{\mathrm{ICRH}}=32.5 \mathrm{MHz}$ where $\% \mathrm{D} \sim 20-30 \%$; the equivalent ${ }^{4} \mathrm{He}-$ $\left({ }^{3} \mathrm{He}\right)-\mathrm{H}$ scenario is applicable for the ITER full field non-active phase with $\mathrm{B}_{\mathrm{T}}=5.3 \mathrm{~T} / \mathrm{f}_{\mathrm{ICRH}}=53 \mathrm{MHz}$ : in this scenario, the $\mathrm{D}$ concentration is adjusted so that the $\mathrm{D} / \mathrm{H}$ 


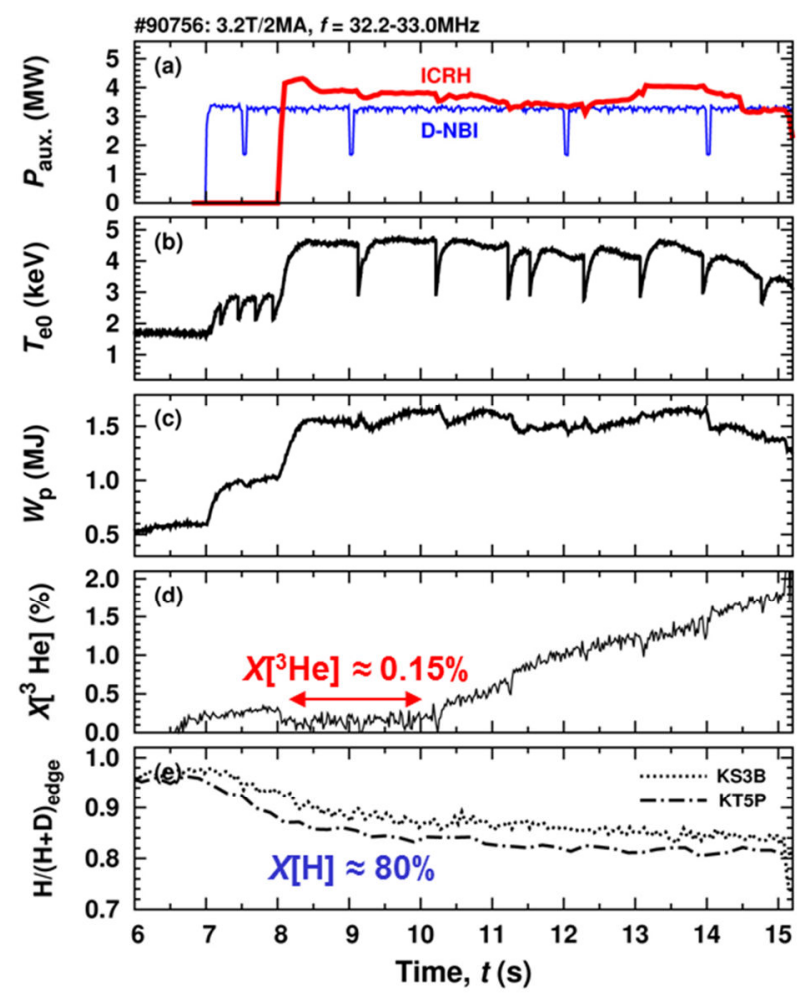

Fig. 10. Reproduced from [32], illustration of the 3 ion scheme heating (a) NBI and ICRH power waveforms, (b) Te, (c) MHD plasma energy, (d ${ }^{3} \mathrm{He}$ concentration and (e) D concentration. In this pulse $+\pi / 2$ strap phasing was used.

hybrid resonance sits close to the $\mathrm{N}=1{ }^{3} \mathrm{He}$ cyclotron resonance layer which, with this combination of $\mathrm{B}_{\mathrm{T}}$ and $\mathrm{f}_{\mathrm{ICRH}}$ is close to the plasma centre. In these conditions, the amplitude and polarisation of the fast wave electric field is favourable for efficient power transfer to ${ }^{3} \mathrm{He}$ ions in tiny concentration (fraction of percent). Fig. 10 shows an example where the 3-ion scheme was used. The heating efficiency was $\sim 70 \%$ and the plasma performance was $\sim 0.15 \mathrm{MJ} / \mathrm{MW}\left(\mathrm{I}_{\mathrm{p}}=1.8 \mathrm{MA}\right.$ and 2 MA). This heating scheme allows creation of a ${ }^{3} \mathrm{He}$ fast population with $\mathrm{MeV}$ energies as was measured with the gamma-ray emission spectroscopy. Using $+\pi / 2$ strap phasing enhanced the efficiency of fast ions generation and plasma heating; this is attributed to a lower $\mathrm{k}_{/ /}$and the inward pinch effect which results in a better confinement of the fast ${ }^{3} \mathrm{He}$ population. We note that the 3-ion heating scheme can also potentially be applied to JET or ITER DT plasmas by choosing an appropriate D$\mathrm{T}$ mix and adjusting $\mathrm{f}_{\mathrm{ICRH}}$ for resonant $\mathrm{Be}$ or fast $\mathrm{T}$ heating [32, 33].

\section{Conclusions and prospects}

ICRH played an important role in the high DD performances recently obtained in JET. Not only does it provide bulk plasma heating but central ICRH is also key for tungsten chase-out and stationarity of discharges. Optimisation of plasma operation to maximize the benefit of ICRH is now part of the scenario development, for example local OMP gas injection to increase antenna coupling is routinely used. Specific experiments were also carried-out to test/tune the ICRH scenarios in preparation for DT operation, or to test some of the heating schemes envisaged for the non-active ITER phase. In particular the dual frequency scheme (H)D and $\left({ }^{3} \mathrm{He}\right) \mathrm{D}$ was tested. In addition, it was confirmed that the (D)H scenario is accessible in an ILW environment and the novel 3-ions ICRH scheme was validated experimentally. One open issue related to the use of ICRH in a metallic environment is the RF enhanced PWI. Experiments were conducted to compare impurity production using antennas with completely different geometries. The ILA produces slightly less high- $Z$ impurities than the A2's and the PWI measured via Be line emission on limiters is in the same ballpark. This will be further studied using antenna and RF-sheath rectification models applied to JET. The availability of the ICRH system and the re-installation of the ILA are two factors that contributed to this progress. Work is ongoing to further improve the RF generators availability and performance, and a Real-Time RF power management system is being implemented to maximise the launched ICRH power when operating close to voltage limits [5]. Remedial work is also in progress to operate the full ILA array, despite the hydraulic fluid leak that developed in the antenna. Ironically, this issue related to the ILA in-vessel matching capacitors validate the choice of external matching elements for ITER.

This work has been carried out within the framework of the EUROfusion Consortium and has received funding from the Euratom research and training programme 2014-2018 under grant agreement No 633053 and from the RCUK Energy Programme [grant number EP/P012450/1]. The views and opinions expressed herein do not necessarily reflect those of the European Commission 'To obtain further information on the data and models underlying this paper please contact PublicationsManager@ukaea.uk.

\section{References}

1. M. Graham et al., Plasma Phys. Control. Fusion 54 074011 (2012)

2. I. Monakhov et al., Nucl. Fusion 53083013 (2013)

3. F. Durodié et al., Plasma Phys. Control. Fusion 54 074012 (2012)

4. P. Dumortier et al., these proceedings

5. I. Monakhov et al., these proceedings

6. P. Jacquet et al., Nucl. Fusion 56046001 (2016)

7. E. Lerche et al., J. Nucl. Mater. 463 634-639 (2015)

8. W. Zhang et al., Nucl. Fusion 57056042 (2017)

9. M. Bécoulet et al., Phys. Plasmas 9 2619-32 (2002)

10. W. Zhang et al., Plasma Phys. Control. Fusion 58 095005 (2016)

11. V. Bobkov et al., Nuclear Materials and Energy, https://doi.org/10.1016/j.nme.2016.10.026

12. V1. Bobkov et al., J. Nucl. Mater. 438 S160-S165 (2013)

13. F. W. Perkins et al., Nucl. Fusion 29 583-92 (1989)

14. L. Colas et al., J. Nucl. Mater. 463 735-738 (2015)

15. C.C Klepper et al., these proceedings

16. C. C Klepper et al., Phys. Scr. T167 014035 (2016) 
17. A. Krivska et al., these proceedings

18. D. Milanesio et al., Nucl. Fusion 49115019 (2009)

19. L. Colas et al., Phys. Plasmas 19092505 (2012)

20. M. Mantsinen et al., these proceedings

21. E. Lerche et al., Nucl. Fusion 56036022 (2016)

22. M. Goniche et al., Plasma Phys. Control. Fusion 59 055001 (2017)

23. D. Gallart et al., 43rd EPS Conf. on Plasma Physics (Leuven, Belgium, 4-8 July) P2.003 (2016)

24. C. Angioni et al., Nucl. Fusion 57056015 (2017)

25. D. Van Eester et al., Proc. 26 ${ }^{\text {th }}$ IAEA-FEC Conference, Kyoto, EX/P6-10

26. M. Schneider et al., these proceedings

27. A. Krasilnikov et al., submitted to Nucl. Fusion

28. E. Lerche et al., AIP Conf. Proc. 1689040003 (2015)

29. P. Mantica et al., Proc. $26^{\text {th }}$ IAEA-FEC Conference, Kyoto, EX/P6-14 (2016)

30. D. Van Eester et al., these proceedings

31. M.-L. Mayoral et al., Nucl. Fusion 46, S550-S563 (2006).

32. Y. Kazakov et al., these proceedings

33. J. Ongena et al., these proceedings 